\title{
Efficient parameter estimation in 2D transport models based on an adjoint formalism
}

\author{
M. Baelmans ${ }^{1}$, J. De Schutter ${ }^{1}$,W. Dekeyser ${ }^{1}$, M. Blommaert ${ }^{2}$, and D. Reiter ${ }^{2}$ \\ ${ }^{1}$ Department of Mechanical Engineering, KU Leuven, Celestijnenlaan 300A, 3001 Leuven, \\ Belgium \\ ${ }^{2}$ Institute for Plasmaphysics (IEK-4), FZ Jülich GmbH, EURATOM Association, Trilateral \\ Euregio Cluster, D-52425 Jülich, Germany
}

\begin{abstract}
2D edge plasma transport simulations are widely used for the interpretation of experimental data in present magnetically confined devices [1]. When analysing available experimental data, estimation of underlying plasma parameters such as anomalous cross field transport coefficients is most often performed by means of a code parameter scan (e.g. in [2] for carbon deposition). Similarly in order to assess the accuracy of predictive (reduced) models, this technique is in use, e.g. in [3]. Vice versa discrepancies between code results based on empirical transport coefficients and experimental data are taken as an indicative measure of the uncertainties [4], thereby implicitly assuming that all other model aspects are correctly describing the underlying physics. Already in $2001 \mathrm{Kim}$ et al. [5] developed an interpretative version of the B2-SOLPS5.0 code using a multi-variable fit procedure to minimize differences between code predictions and experimental measurements. Transport coefficients and the fractional amount of neutrals pumped were varied to achieve best matches to individual experiments. Via regression techniques scaling laws for transport coefficients were deduced.

In this contribution we employ the adjoint formalism for gradient calculations to speed up both parameter estimation and uncertainty assessment when a larger set of parameters (including spatially or temporal varying coefficients) is involved. Such "dual" techniques are well-known for linear problems. It is recently applied for the thermal characterization of the surface carbon layer in JET [6]. The adjoint approach is equally well suited for non-linear sets of partial differential equations as encountered e.g. in $2 \mathrm{D}$ edge plasma transport studies. After setting up the adjoint equations, the gradient of the objective functional w.r.t. the control variables can be deduced. The optimization problem for the underlying transport parameters is then solved with a BFGS algorithm with inexact line search. The method is illustrated by means of a test case where radial ion as well as neutral transport coefficients are deduced from prescribed midplane and target density and temperature profiles. In addition, first results for a test case with typical JET parameters will be addressed.
\end{abstract}

\section{References}

[1] D. Reiter et al., Fusion Science and Technology 47 (2005), 172-186.

[2] A. Xuereb et al., Journal of Nuclear Materials 396 (2010), 228-233 .

[3] D. Coster et al., Physica Scripta. Vol. T108(2004), 7-13.

[4] V. Kotov et al., Plasma Phys. Control. Fusion 50 (2008), doi:10.1088/0741-3335/50/10/105012.

[5] J.-W. Kim et al., J. of Nucl. Mat.290-293 (2001) 644-647.

[6] J.Gaspar et al., Int. Journal of Heat and Mass Transfer 56 (2013) 147-157.

Corresponding author: M. Baelmans, tine.baelmans@kuleuven.be 